\title{
CONVERGENCE OF DIAGONAL PADÉ APPROXIMANTS FOR FUNCTIONS ANALYTIC NEAR 0
}

\author{
D. S. LUBINSKY
}

\begin{abstract}
For functions analytic in a neighbourhood of 0 , we show that at least for a subsequence of the diagonal Padé approximants, the point 0 attracts a zero proportion of the poles. The same is true for every "sufficiently dense" diagonal subsequence. Consequently these subsequences have a convergence in capacity type property, which is possibly the correct analogue of the NuttallPommerenke theorem in this setting.
\end{abstract}

\section{INTRODUCTION}

Recall that if $f$ is analytic near 0 , then for $m, n \geq 0$, the $m, n$ Padé approximant to $f$ is a rational function $[m / n](z)=(P / Q)(z)$, where $P, Q$ have degree $\leq m, n$ respectively, $Q$ is not identically zero, and

$$
(f Q-P)(z)=O\left(z^{m+n+1}\right), \quad z \rightarrow 0 .
$$

For functions meromorphic in $\mathbb{C}$, or even with singularities of capacity 0 , it is known that the diagonal sequence $\{[n / n]\}_{n=1}^{\infty}$ converges in capacity and in measure $[11,14]$. Similar results are available in more general circumstances $[3,6,16,17,19]$.

By contrast, for functions analytic only near 0 , the full diagonal sequence of Padé approximants need not converge in capacity in any neighbourhood of zero $[7,8,15]$, and moreover, at least for infinitely many $n,[n / n]$ may have at least $n-\log n$ poles arbitrarily near 0 [18]. (We could replace $\log n$ by any sequence increasing to $\infty$.) The 1961 Baker-Gammel-Wills conjecture $[1,2]$ asserts that a subsequence of $\{[n / n]\}$ converges uniformly near 0 , but at present it is not even known if a subsequence converges in capacity.

In this paper we show that, at least for a subsequence of $\{[n / n]\}$, the proportion of poles of $[n / n]$ near 0 shrinks to 0 , in a certain sense. This result also holds for subsequences of $\left\{\left[n_{j} / n_{j}\right]\right\}$ provided $n_{j+1} / n_{j} \rightarrow 1$ as $j \rightarrow \infty$. Then we deduce a convergence in capacity type property. Since by a variable scaling $z \rightarrow r z$ any function analytic near 0 can be scaled to a function analytic in $|z|<1$, the transformation properties of Padé approximants permit us to consider only the latter:

Received by the editors June 2,1994; originally communicated to the Proceedings of the AMS by J. Marshall Ash.

1991 Mathematics Subject Classification. Primary 41A21, 30E10.

Key words and phrases. Padé approximants, convergence of diagonal Padé approximants, pole distribution. 
Theorem 1.1. Let $f$ be analytic in $\{z:|z|<1\}$. Let $\left\{n_{j}\right\}_{j=1}^{\infty}$ be an increasing sequence of positive integers with

$$
\lim _{j \rightarrow \infty} n_{j+1} / n_{j}=1
$$

Let $0<\delta<1$. Then there exists an infinite sequence of positive integers $\mathscr{S}$ with the following property: For $j \in \mathscr{S}$, the total multiplicity of poles of $\left[n_{j} / n_{j}\right]$ in $\left\{z:|z| \leq e^{-19 / \delta}\right\}$ is at most $\delta n_{j}$.

Under a regularity assumption on the errors of best rational approximation, we can say the same for full sequences of Padé approximants: For $0<\rho<1$, we let

$$
E_{n n}(f ; \rho):=\inf \left\{\|f-R\|_{L_{\infty}(|z| \leq \rho)}: R \text { is a rational function of type }(n, n)\right\} \text {. }
$$

Theorem 1.2. Assume that $f$ is analytic in $\{z:|z|<1\}$, and that

$$
0<\limsup _{n \rightarrow \infty} E_{n n}(f ; \rho)^{1 / n} \leq \kappa(\rho) \liminf _{n \rightarrow \infty} E_{n n}(f ; \rho)^{1 / n},
$$

where $\kappa(\rho)$ is finite for $\rho \in(0,1)$. Then for large enough $n$, the total multiplicity of poles of $[n / n](z)$ in $\{z:|z| \leq \rho\}$ is at most $\delta n$, provided

$$
\rho \kappa(\rho)^{1 / \delta} \leq \exp (-19 / \delta) .
$$

In particular, if $\lim _{n \rightarrow \infty} E_{n n}(f ; \rho)^{1 / n}$ exists for $0<\rho<1$, then for $n$ large enough, the total multiplicity of poles of $[n / n](z)$ in $\{z:|z| \leq \exp (-19 / \delta)\}$ is at most $\delta n$.

Remarks. (i) Similar results hold if we consider sectorial sequences of Padé approximants of the form $\left\{\left[m_{j} / n_{j}\right]\right\}$, where $\left\{m_{j}\right\},\left\{n_{j}\right\}$ satisfy (1.1) and, for some fixed $\lambda$,

$$
1 / \lambda \leq m_{j} / n_{j} \leq \lambda, \quad j \geq 1 \text {. }
$$

The formulation will be more complicated and the proofs will be messier, but we hope to attend to this in a subsequent paper. (See [9], where similar results were proved for functions analytic in $\mathbb{C}$ except for singularities of capacity 0 and for general sectorial sequences $\left\{\left[m_{j} / n_{j}\right]\right\}$.)

(ii) Note that the size of the neighbourhood in which there are at most $\delta n$ poles is a function of $\delta$ only, not of $f$. However, the factor $e^{-19}$ is not optimal.

(iii) Note that if

$$
\liminf _{n \rightarrow \infty} E_{n n}(f ; \rho)^{1 / n}=0,
$$

then it is easy to see that a subsequence of the $[n / n]$ Padé approximants actually converges in capacity on compact subsets of $\{z:|z|<\rho\}$. Note too that if

$$
\lim _{n \rightarrow \infty} E_{n n}(f ; \rho)^{1 / n}=0,
$$

then $f$ belongs to the Gončar-Walsh class $[3,10]$, the $[n / n]$ Pade approximants converge in capacity in $\{z:|z|<1\}[3,19]$, and for $j \in \mathscr{S}$ all but $o\left(n_{j}\right)$ poles of $\left[n_{j} / n_{j}\right]$ leave every compact subset of $\{z:|z|<1\}[9,10]$.

Now we turn to convergence in capacity. Recall that, for a compact set $K$, the logarithmic capacity $\operatorname{cap}(K)$ is defined by

$$
\operatorname{cap}(K):=\lim _{n \rightarrow \infty}\left(\min _{P_{n}}\left\|P_{n}\right\|_{L_{x}(K)}\right)^{1 / n},
$$


where the minimum is taken over all monic polynomials $P_{n}$ of degree $n$. For arbitrary $S$, the inner logarithmic capacity $\operatorname{cap}(S)$ is defined by

$$
\operatorname{cap}(S):=\sup \{\operatorname{cap}(K): K \subset S, K \text { compact }\} \text {. }
$$

Convergence in capacity is essentially the same as convergence in measure. We say $f_{n} \rightarrow f$ in capacity in $\{z:|z| \leq r\}$ if $\forall \varepsilon>0$,

$$
\operatorname{cap}\left\{z:|z| \leq r \text { and }\left|f-f_{n}\right|(z) \leq \varepsilon\right\} \rightarrow 0, \quad n \rightarrow \infty .
$$

The Nuttall-Pommerenke theorem [11, 14] and its extensions actually prove geometric convergence in capacity under suitable hypotheses on $f$ :

$$
\operatorname{cap}\left\{z:|z| \leq r \text { and }|f-[n / n]|(z) \geq \varepsilon^{n}\right\} \rightarrow 0, \quad n \rightarrow \infty .
$$

Here we shall show that Theorem 1.1 implies a weak convergence in capacity property:

Given $0<\Delta<\frac{1}{2}, A>1$, there exists $\rho=\rho(A)<1$ (independent of $f$ ) such that, for all $n$ in a subsequence,

$$
\operatorname{cap}\left\{z:|z| \leq \rho:|f-[n / n]|(z) \geq \rho^{n \Delta}\right\} \leq \operatorname{cap}\{z:|z| \leq \rho\}^{A}=\rho^{A} .
$$

The same estimate holds if we replace cap by planar Lebesgue measure or onedimensional Hausdorff content.

The point is that, in most of $\{z:|z| \leq \rho\},[n / n]$ is geometrically close to $f$, and we have a weak convergence in capacity property: The capacity (or area or one-dimensional Hausdorff content) of the set on which $[n / n]$ does not approximate is an arbitrarily small proportion of the total capacity (or area or content). I believe that in the setting of the following theorem the conclusion of Theorem 1.3 may possibly be the correct analogue of the Nuttall-Pommerenke theorem: Nothing more can be said of subsequences of $\left\{\left[n_{j} / n_{j}\right]\right\}$, other than this weak convergence in capacity type property near 0 . Of subsequences of the full diagonal sequence $\{[n / n]\}$, the Baker-Gammel-Wills conjecture may well be true.

Theorem 1.3. Let $f$ be analytic in $\{z:|z|<1\}$. Let $0<\Delta<\frac{1}{2}, A>1$ and $\rho:=\frac{1}{2} \exp \left(-19 A /\left(\frac{1}{2}-\Delta\right)\right)$. Let $\left\{n_{j}\right\}_{j=1}^{\infty}$ be an increasing sequence of positive integers satisfying (1.1). Then there exists an infinite sequence of positive integers $\mathscr{S}$ with the following property: For $j \in \mathscr{S}$,

$$
\operatorname{cap}\left\{z:|z| \leq \rho \text { and }\left|f-\left[n_{j} / n_{j}\right]\right|(z)>\left(\frac{|z|}{\rho} \cdot \rho^{\Delta}\right)^{2 n_{j}}\right\} \leq \operatorname{cap}\{z:|z| \leq \rho\}^{A} \text {. }
$$

Remarks. (i) The restriction $\Delta<\frac{1}{2}$ is related to the exponent $\frac{1}{2}$ in the righthand side of

$$
\limsup _{n \rightarrow \infty} E_{n n}(f ; \rho)^{1 /(2 n)} \leq \rho^{1 / 2}
$$

It is now known [12] that

$$
\liminf _{n \rightarrow \infty} E_{n n}(f ; \rho)^{1 /(2 n)} \leq \rho .
$$

Consequently, if we assume that $\lim _{n \rightarrow \infty} E_{n n}(f ; \rho)^{1 /(2 n)}$ exists for $0<\rho<1$, then our proof allows us to replace $0<\Delta<\frac{1}{2}$ by $0<\Delta<1$ and $\rho=$ $\frac{1}{2} \exp \left(-19 A /\left(\frac{1}{2}-\Delta\right)\right)$ by $\rho=\frac{1}{2} \exp (-19 A /(1-\Delta))$ in the above result. In 
that case also, the weak convergence in capacity will hold for the full diagonal sequence, and not just a subsequence.

(ii) The subsequence $\mathscr{S}$ in Theorem 1.3 is the same sequence as in Theorem 1.1 , with a suitable choice of $\delta=\delta(A)$.

We prove Theorems 1.1 and 1.2 in $\S 2$ and Theorem 1.3 in $\S 3$.

\section{Proof of Theorems 1.1 and 1.2}

We shall use the notion of one-dimensional Hausdorff content:

$$
m(E):=\inf \left\{\sum_{j} \operatorname{diam}\left(B_{j}\right): E \subset \bigcup_{j} B_{j}\right\},
$$

where the inf is taken over all countable collections of balls $\left\{B_{j}\right\}$ of diameters \{diam $\left.B_{j}\right\}$ covering $E$. We first present four lemmas (at least two of which are standard), and then prove Theorems 1.2 and 1.1. Throughout $\mathscr{P}_{n}$ denotes the polynomials of degree $\leq n$, and $C, C_{1}, C_{2}, \ldots$ denote constants independent of $n, P$, and $z$. The same symbol does not necessarily denote the same constant in different occurrences. In the sequel, $[n / n]=p_{n} / q_{n}$.

Lemma 2.1. Let $U \in \mathscr{P}_{l} \backslash\{0\}$ and $0<\varepsilon \leq \rho$. Then there exists a set $\mathscr{E} \subset[0, \rho]$ such that $m(\mathscr{E}) \leq \varepsilon$ and, for $\sigma \in[0, \rho] \backslash \mathscr{E}$,

$$
\max \{|U(t) / U(z)|:|t|=\rho,|z|=\sigma\} \leq(12 e \rho / \varepsilon)^{l} .
$$

Proof. Split $U=c V W$, where $c \neq 0$, and $V, W$ are monic polynomials of degree $\nu, \omega$, respectively, with zeros outside $|z| \leq 2 \rho$, inside $|z| \leq 2 \rho$, respectively. Now for $|a| \geq 2 \rho,|t|=\rho,|z| \leq \rho$,

$$
\left|\frac{t-a}{z-a}\right| \leq \frac{1+|t / a|}{1-|z / a|} \leq 3
$$

We deduce that

$$
|V(t) / V(z)| \leq 3^{\nu}, \quad|t|=\rho,|z| \leq \rho .
$$

Next, by Cartan's lemma [1, p. 174],

$$
|W(z)| \geq(\varepsilon / 4 e)^{\omega}, \quad z \in \mathbb{C} \backslash \mathscr{F},
$$

where $m(\mathscr{F}) \leq \varepsilon$. Then using an easy covering argument, we see that $\mathscr{E}:=$ $\{|z|: z \in \mathscr{F}\}$ also has $m(\mathscr{E}) \leq \varepsilon$. Moreover for $|t|=\rho$,

$$
|W(t)| \leq(3 \rho)^{\omega} .
$$

These last two inequalities and (2.2) give (2.1).

Lemma 2.2. Let $f$ be analytic in $\{z:|z|<1\}$. Let $0<\varepsilon \leq \rho<1$. There exists $\mathscr{E}_{n}$ with $m\left(\mathscr{E}_{n}\right) \leq \varepsilon$, such that, for $\sigma \in[0, \rho] \backslash \mathscr{E}_{n}$,

$$
\max _{|z|=\sigma}|f-[n / n](z)| \leq E_{n n}(f ; \rho)\left(\frac{12 e \sigma}{\varepsilon}\right)^{2 n} \frac{\sigma}{\rho-\sigma} .
$$

In particular, for some $\rho_{1} \in\left[\frac{1}{3} \rho, \frac{2}{3} \rho\right]$,

$$
\max _{|z|=\rho_{1}}|f-[n / n]|(z) \leq 2 E_{n n}(f ; \rho)(32 e)^{2 n} \text {. }
$$


Proof. Let $r_{n}^{*}:=p_{n}^{*} / q_{n}^{*}$ be the best approximant of type $(n, n)$ to $f$ on $|z| \leq$ $\rho$. Then for $|z|<\rho$,

$$
q_{n}^{*}(z)\left(f q_{n}-p_{n}\right)(z) / z^{2 n+1}=\frac{1}{2 \pi i} \int_{|t|=\rho}\left[q_{n}(t)\left(f q_{n}^{*}-p_{n}^{*}\right)(t) / t^{2 n+1}\right] \frac{d t}{t-z} .
$$

This is an easy consequence of Cauchy's integral formula and the fact that, for any $\Pi \in \mathscr{P}_{2 n}$,

$$
\frac{1}{2 \pi i} \int_{|t|=\rho}\left[\Pi(t) / t^{2 n+1}\right] \frac{d t}{t-z}=0 .
$$

(We chose $\Pi:=p_{n}^{*} q_{n}-p_{n} q_{n}^{*}$.) We deduce that, for $\sigma<\rho$,

$$
\begin{aligned}
\max _{|z|=\sigma}|f-[n / n](z)| & \\
\leq & \left(\frac{\sigma}{\rho}\right)^{2 n+1} \max \left\{\left|\frac{\left(q_{n} q_{n}^{*}\right)(t)}{\left(q_{n} q_{n}^{*}\right)(z)}\right|:|t|=\rho,|z|=\sigma\right\} \frac{\rho}{\rho-\sigma} E_{n n}(f ; \rho) \\
& \leq\left(\frac{12 e \sigma}{\varepsilon}\right)^{2 n} \frac{\sigma}{\rho-\sigma} E_{n n}(f ; \rho),
\end{aligned}
$$

by Lemma 2.1 , provided $\sigma \notin \mathscr{E}$, where $m(\mathscr{E}) \leq \varepsilon$. In particular, if $\varepsilon=\rho / 4$, we can choose such a $\rho_{1}:=\sigma \in\left[\frac{1}{3} \rho, \frac{2}{3} \rho\right] \backslash \mathscr{E}$.

We shall need a lemma of Gončar and Grigorjan:

Lemma 2.3. If $g$ is analytic in $\{z:|z| \leq \rho\}$ except for poles of total multiplicity $m$, none lying on $|z|=\rho$, and if $\mathscr{A}_{\rho}(g)$ denotes the analytic part of $g$ in $\{z:|z| \leq \rho\}$ (that is, $g$ minus its principal parts in $|z|<\rho)$, then

$$
\left\|\mathscr{A}_{\rho}(g)\right\|_{L_{\infty}(|z| \leq \rho)} \leq 7 m^{2}\|g\|_{L_{\infty}(|z|=\rho)} .
$$

Proof. See [4]. For more precise results and references, see [5, 12, 13].

Following is our main lemma:

Lemma 2.4. Let $f$ be analytic in $\{z:|z|<1\}$, and $0<\rho<1, K \geq 1$, with $3 K \rho<1$. If $[n / n]$ has $\tau=\tau(n)$ poles counting multiplicity in $\{z:|z| \leq K \rho\}$, then, for large enough $n$,

$$
E_{n-\tau, n-\tau}(f ; K \rho) \leq\left[e^{16} K\right]^{n} E_{n n}(f ; \rho)^{1+(\log 6 K) /(\log 3 K \rho)} .
$$

Proof. Let $S_{m}$ be the $m$ th partial sum of the Maclaurin series of $f$. Let $\varepsilon:=3 K \rho$. We have, for large enough $m$,

$$
\left\|f-S_{m}\right\|_{L_{\infty}(|z| \leq 2 K \rho)} \leq \varepsilon^{m} .
$$

Let $\langle x\rangle$ denote the largest integer $\leq x$. We let

$$
m:=\left\langle\frac{\log E_{n n}(f ; \rho)}{\log \varepsilon}\right\rangle+1,
$$

so that $\varepsilon^{m} \leq E_{n n}(f ; \rho)$. Let $\rho_{1} \in\left[\frac{1}{3} \rho, \frac{2}{3} \rho\right]$ be as in Lemma 2.2. We deduce from (2.4) and our choice of $m$ that, for $n$ large enough,

$$
\left\|S_{m}-[n / n]\right\|_{L_{\infty}\left(|z|=\rho_{1}\right)} \leq E_{n n}(f ; \rho)\left\{1+2(32 e)^{2 n}\right\},
$$

so

$$
\left\|S_{m} q_{n}-p_{n}\right\|_{L_{\infty}\left(|z|=\rho_{1}\right)} \leq E_{n n}(f ; \rho) 3(32 e)^{2 n}\left\|q_{n}\right\|_{L_{\infty}\left(|z|=\rho_{1}\right)} .
$$


The Bernstein-Walsh lemma gives

$$
\left\|S_{m} q_{n}-p_{n}\right\|_{L_{\infty}(|z| \leq 2 K \rho)} \leq E_{n n}(f ; \rho) 3(32 e)^{2 n}\left\|q_{n}\right\|_{L_{\infty}\left(|z|=\rho_{1}\right)}(6 K)^{m+n} \text {. }
$$

We deduce that, for $\sigma \leq 2 K \rho$,

$$
\begin{aligned}
\| f- & {[n / n]\left\|_{L_{\infty}(|z|=\sigma)} \leq\right\| f-S_{m}\left\|_{L_{\infty}(|z|=\sigma)}+\right\| S_{m}-[n / n] \|_{L_{\infty}(|z|=\sigma)} } \\
\leq & E_{n n}(f ; \rho)\left[1+3(32 e)^{2 n}(6 K)^{m+n} \max \left\{\left|\frac{q_{n}(t)}{q_{n}(z)}\right|:|t|=\rho_{1},|z|=\sigma\right\}\right],
\end{aligned}
$$

provided, of course, that the right-hand side is finite. By Lemma 2.1 (with $\rho$ there replaced by $2 K \rho$, and $\left.\varepsilon=\frac{3}{4} K \rho\right)$, we can choose $\sigma_{1} \in(K \rho, 2 K \rho)$ such that

$$
\begin{aligned}
\max & \left\{\left|\frac{q_{n}(t)}{q_{n}(z)}\right|:|t|=\rho_{1},|z|=\sigma_{1}\right\} \leq \max \left\{\left|\frac{q_{n}(t)}{q_{n}(z)}\right|:|t|=2 K \rho,|z|=\sigma_{1}\right\} \\
& \leq\left(\frac{12 e \cdot 2 K \rho}{3 K \rho / 4}\right)^{n}=(32 e)^{n} .
\end{aligned}
$$

Since $f$ is analytic, $\mathscr{A}_{\sigma_{1}}(f-[n / n])=f-\mathscr{A}_{\sigma_{1}}([n / n])$. Also $\sigma_{1} \geq K \rho$. Then Lemma 2.3 gives

$$
\begin{aligned}
\| f- & \mathscr{A}_{\sigma_{1}}([n / \cdot n])\left\|_{L_{\infty}(|z| \leq K \rho)} \leq\right\| f-\mathscr{A}_{\sigma_{1}}([n / n]) \|_{L_{\infty}\left(|z|=\sigma_{1}\right)} \\
& \leq 7 n^{2}\|f-[n / n]\|_{L_{\infty}\left(|z|=\sigma_{1}\right)} \leq 28 n^{2}\left[6 K(32 e)^{3}\right]^{n}(6 K)^{m} E_{n n}(f ; \rho) \\
& \leq E_{n n}(f ; \rho)^{1+\log 6 K / \log 3 K \rho}\left(e^{16} K\right)^{n},
\end{aligned}
$$

for $n$ large enough, by our choice of $m$ and of $\varepsilon=3 K \rho$. Since $[n / n]$ has at least $\tau$ poles in $|z| \leq K \rho<\sigma_{1}, \mathscr{A}_{\sigma_{1}}([n / n])$ is a rational function of type $(n-\tau, n-\tau)$, and the result follows.

We turn to the proofs of the theorems. To indicate the ideas, we first prove the simpler Theorem 1.2. In the sequel, we let

$$
A(\rho):=\limsup _{n \rightarrow \infty} E_{n n}(f ; \rho)^{1 / n} .
$$

Recall (as in the Introduction) that if $A(\rho)=0$ for some $\rho \in(0,1)$, then, by a result of Gončar [3], $A(\rho)=0$ for all $0<\rho<1$, and then stronger results are available [9]. So we assume that $A(\rho)>0$ for all $\rho>0$ in the sequel.

Proof of Theorem 1.2. Assume that, for some $\delta \in(0,1), \rho \in\left(0, \frac{1}{2}\right)$, and for $n$ belonging to some infinite sequence of integers $\mathscr{N},[n / n]$ has poles of total multiplicity $\geq \delta n$ in $\{z:|z| \leq \rho\}$. We show that $\rho$ cannot be too small assuming that $\mathscr{N}$ is an infinite set. Applying Lemma 2.4 with $K=1$ gives

$$
E_{\langle n(1-\delta)\rangle,\langle n(1-\delta)\rangle}(f ; \rho) \leq e^{16 n} E_{n n}(f ; \rho)^{1+\log 6 / \log 3 \rho} .
$$

Taking $n$th roots, letting $n \rightarrow \infty$ through $\mathscr{N}$, and using (1.2) gives

$$
\left[\kappa(\rho)^{-1} A(\rho)\right]^{1-\delta} \leq e^{16} A(\rho)^{1+\log 6 / \log 3 \rho} .
$$

That is,

$$
A(\rho)^{-\delta-\log 6 / \log 3 \rho} \leq e^{16} \kappa(\rho) .
$$

(Recall that $(1.2)$ forces $\kappa(\rho) \geq 1$.) The exponent of $A(\rho)$ is negative if

$$
\rho \leq \frac{1}{3} \exp \left(-\frac{\log 6}{\delta}\right)\left(\leq \frac{1}{18}\right) \text {. }
$$


Now $A(\rho) \leq \rho$ by analyticity of $f$ in $|z|<1$, so, for $\rho$ satisfying (2.7), it follows from (2.6) that

$$
\delta|\log \rho| \leq 16+\log \kappa(\rho)+\log 6\left|\frac{\log \rho}{\log 3 \rho}\right| .
$$

Here for $\rho \leq \frac{1}{18}$,

$$
\log 6\left|\frac{\log \rho}{\log 3 \rho}\right| \leq \log 18<3
$$

So we obtain

$$
\rho \kappa(\rho)^{1 / \delta}>\exp (-19 / \delta) .
$$

Therefore for large enough $n,[n / n]$ can have no more than $\delta n$ poles in $\{z:|z|<\rho\}$ if $\rho \kappa(\rho)^{1 / \delta} \leq \exp (-19 / \delta)$.

Proof of Theorem 1.1. The consequence of (1.1) that we shall use is

$$
\underset{k \rightarrow \infty}{\limsup } E_{n_{k}, n_{k}}(f ; \rho)^{1 / n_{k}}=\limsup _{n \rightarrow \infty} E_{n n}(f ; \rho)^{1 / n}=A(\rho) .
$$

This follows easily from the fact that $E_{n n}(f ; \rho)$ is decreasing in $n$. Let $0<$ $\eta<\delta<1$. For large enough $k$, we define $j=j(k)$ to be the largest integer $j$ for which $n_{k} \geq n_{j}(1-\eta)$, so that

$$
n_{j(k)}(1-\eta) \leq n_{k}<n_{j(k)+1}(1-\eta) \text {. }
$$

Let $\tau_{k}:=n_{j(k)}-n_{k}$. We see from (1.1) and our choice of $j(k)$ that

$$
\lim _{k \rightarrow \infty} \tau_{k} / n_{j(k)}=\eta ; \quad \lim _{k \rightarrow \infty} n_{k} / n_{j(k)}=1-\eta .
$$

Suppose that for some $0<\rho<1$ and for large enough $k,\left[n_{k} / n_{k}\right]$ has more than $\delta n_{k}$ poles in $\{z:|z| \leq \rho\}$. Then for large enough $k$, and $j=j(k)$, $\left[n_{j(k)} / n_{j(k)}\right]$ has $>\delta n_{j(k)}>\tau_{k}$ poles in $\{z:|z| \leq \rho\}$ (recall that $\eta<\delta$ ). As $n_{j(k)}-\tau_{k}=n_{k}$, Lemma 2.4 (with $K=1$ ) gives

$$
E_{n_{k}, n_{k}}(f ; \rho) \leq e^{16 n_{j(k)}} E_{n_{j(k)}, n_{j(k)}}(f ; \rho)^{1+(\log 6) /(\log 3 \rho)} .
$$

Taking $n_{j(k)}$ th roots in this last inequality, and then lim sups as $k \rightarrow \infty$, and using (2.8) and (2.9), give

$$
A(\rho)^{1-\eta} \leq e^{16} A(\rho)^{1+(\log 6) /(\log 3 \rho)} .
$$

Since $\eta<\delta$ is arbitrary, we deduce that

$$
A(\rho)^{1-\delta} \leq e^{16} A(\rho)^{1+(\log 6) /(\log 3 \rho)} .
$$

Then

$$
A(\rho)^{-\delta-(\log 6) /(\log 3 \rho)} \leq e^{16}
$$

This is the exact same relation as (2.6) with $\kappa(\rho) \equiv 1$. Proceeding exactly as in the previous proof with $\kappa(\rho) \equiv 1$, we obtain

$$
\rho>\exp (-19 / \delta) \text {. }
$$

\section{Proof of Theorem 1.3}

Let $0<\rho<\frac{1}{2}, 0<\delta<1, A>1$, and assume that for $n$ belonging to some infinite sequence of integers $\mathcal{N},[n / n]=p_{n} / q_{n}$ has no more than $\delta n$ 
poles, counting multiplicity, in $\{z:|z| \leq 2 \rho\}$. Let $r_{n}^{*}:=p_{n}^{*} / q_{n}^{*}$ be a best approximation of type $(n, n)$ to $f$ on $\{z:|z| \leq 2 \rho\}$. We begin with the identity from Lemma 2.2: For $|z|<2 \rho$,

$$
(f-[n / n])(z)=\frac{1}{2 \pi i} \int_{|t|=2 \rho}\left(\frac{z}{t}\right)^{2 n+1} \frac{\left(q_{n}^{*} q_{n}\right)(t)}{\left(q_{n}^{*} q_{n}\right)(z)} \frac{\left(f-r_{n}^{*}\right)(t)}{t-z} d t .
$$

We deduce that, for $|z| \leq \rho$,

$$
|f-[n / n]|(z) \leq 2\left(\frac{|z|}{2 \rho}\right)^{2 n} E_{n n}(f ; 2 \rho) \max _{|t|=2 \rho}\left|\frac{\left(q_{n}^{*} q_{n}\right)(t)}{\left(q_{n}^{*} q_{n}\right)(z)}\right| .
$$

Now for $n \geq n_{0}(\rho)$,

$$
E_{n n}(f ; 2 \rho) \leq(3 \rho)^{n} .
$$

Recall that $q_{n}^{*}$ has all zeros outside $|z| \leq 2 \rho$. We split $q_{n}=S_{n} U_{n}$, where $S_{n}$ is monic of degree $s_{n} \leq \delta n$ and has zeros in $|z| \leq 2 \rho$, and $U_{n}$ has zeros in $|z|>2 \rho$. Exactly as in the proof of Lemma 2.1 , we see that, for $|z| \leq \rho$,

$$
\max _{|t|=\rho}\left|\frac{\left(q_{n}^{*} U_{n}\right)(t)}{\left(q_{n}^{*} U_{n}\right)(z)}\right| \leq 3^{2 n}
$$

Next, as $S_{n}$ is monic, the set $\mathscr{E}_{n}:=\left\{z:\left|S_{n}(z)\right| \leq \rho^{A s_{n}}\right\}$ has $\operatorname{cap}\left(\mathscr{C}_{n}\right)=\rho^{A}$. Then as in Lemma 2.1 , since $S_{n}$ has all its zeros in $|u| \leq 2 \rho$, we have, for $|z| \leq \rho, z \notin \mathscr{E}_{n}$,

$$
\max _{|t|=\rho}\left|\frac{\left(S_{n}\right)(t)}{\left(S_{n}\right)(z)}\right| \leq\left(\frac{3 \rho}{\rho^{A}}\right)^{S_{n}} \leq\left(3 \rho^{-A}\right)^{\delta n} .
$$

Combining (3.1)-(3.4), we have, for $|z| \leq \rho, z \notin \mathscr{C}_{n}$,

$$
|f-[n / n]|(z)^{1 /(2 n)} \leq 2^{1 / 2 n} \frac{|z|}{2 \rho}(3 \rho)^{1 / 2} 3\left(3 \rho^{-A}\right)^{\delta / 2} \leq \frac{|z|}{\rho} 8 \rho^{(1-A \delta) / 2} \leq \frac{|z|}{\rho} \rho^{\Delta},
$$

provided

$$
\rho^{1 / 2-\Delta-A \delta / 2} \leq \frac{1}{8}
$$

In summary, we have shown that, for large enough $n \in \mathscr{N}$,

$$
\begin{gathered}
\operatorname{cap}\left\{z:|z| \leq \rho \text { and }|f-[n / n]|(z)^{1 / 2 n}>\frac{|z|}{\rho} \rho^{\Delta}\right\} \leq \operatorname{cap}\left(\mathscr{E}_{n}\right) \\
=\rho^{A}=\operatorname{cap}\{z:|z| \leq \rho\}^{A},
\end{gathered}
$$

provided (3.5) holds. Let us choose $\delta$ and $\rho$ by

$$
A \delta=\frac{1}{2}-\Delta, \quad 2 \rho=\exp (-19 / \delta)=\exp \left(-19 A /\left(\frac{1}{2}-\Delta\right)\right) .
$$

Then

$$
\rho^{1 / 2-\Delta-A \delta / 2}=\rho^{(1 / 2-\Delta) / 2} \leq \exp (-19 A / 2) \leq \exp (-19 / 2),
$$

as $A \geq 1$, so (3.5) is satisfied. Finally, with this choice of $\delta$ and $\rho$, Theorem 1.1 guarantees that, given $\left\{n_{j}\right\}$ satisfying (1.1), we can find infinitely many $j$ such that for $n=n_{j}, j \in \mathscr{S}$; that is, $\left[n_{j} / n_{j}\right]$ has at most $\delta n_{j}$ poles in $\{z:|z| \leq 2 \rho\}$. 
We remark that when the limit

$$
\lim _{n \rightarrow \infty} E_{n n}(f ; \rho)^{1 / n}
$$

exists, then the aforementioned result of Parfenov guarantees that it is $\leq \rho^{2}$. Then we can replace (3.2) by

$$
E_{n n}(f ; 2 \rho) \leq\left(3 \rho^{2}\right)^{n}
$$

Proceeding as before, we see that we can then choose $\rho=\frac{1}{2} \exp (-19 A /(1-\Delta))$, for any $0<\Delta<1$.

\section{REFERENCES}

1. G. A. Baker, Essentials of Padé approximants, Academic Press, New York, 1975.

2. G. A. Baker and P. R. Graves-Morris, Padé approximants. Part 1: Basic theory, Encyclopaedia of Mathematics and its Applications, Vol. 13, Addison-Wesley, Reading, MA, 1981.

3. A. A. Gončar, A local condition of single-valuedness of analytic functions, Math. USSR Sb. 18 (1972), 151-167.

4. A. A. Gončar and L. D. Grigorjan, On estimates of the norm of the holomorphic component of a meromorphic function, Math. USSR Sb. 28 (1976), 571-575.

5. L. D. Grigorjan, Estimates of the norm of the holomorphic components of functions meromorphic in domains with a smooth boundary, Math. USSR Sb. 29 (1976), 139-146.

6. J. Karlsson, Rational interpolation and best rational approximation, J. Math. Anal. Appl. 53 (1976), 38-52.

7. D. S Lubinsky, Diagonal Padé approximants and capacity, J. Math. Anal. Appl. 78 (1980), 58-67.

8. _ Divergence of complex rational approximations, Pacific J. Math. 108 (1983), 141-153.

9. - Distribution of poles of diagonal rational approximants to functions of fast rational approximability, Constr. Approx. 7 (1991), 501-519.

10. $\ldots$ Spurious poles in diagonal rational approximation, Progress in Approximation Theory: An International Perspective (A. A. Gončar and E. B. Saff, eds.), Springer Ser. Comput. Math., vol. 9, Springer-Verlag, New York, 1992, pp. 191-213.

11. J. Nuttall, The convergence of Padé approximants of meromorphic functions, J. Math. Anal. Appl. 31 (1970), 147-153.

12. O. G. Parfenov, Estimates of the singular numbers of the Carleson imbedding operator, Math. USSR Sb. 59 (1988), 497-514.

13. A. A. Pekarskii, Best rational approximations in the complex domain, Proc. Steklov. Inst. Math. 190 (1992), 231-243.

14. Ch. Pommerenke, Padé approximants and convergence in capacity, J. Math. Anal. Appl. 41 (1973), 775-780.

15. E. A. Rahmanov, On the convergence of Padé approximants in classes of holomorphic functions, Math. USSR Sb. 40 (1981), 149-155.

16. H. Stahl, A note on three conjectures by Gonchar on rational approximation, J. Approx. Theory 50 (1987), 3-7.

17. _ General convergence results for Padé approximants, Approximation Theory VI (C. K. Chui et al., eds.), Academic Press, San Diego, CA, 1989, pp. 605-634.

18. $\mathrm{H}$. Wallin, The convergence of Padé approximants and the size of the power series coefficients, Applicable Anal. 4 (1974), 235-251.

19. $\ldots$ Potential theory and approximation of analytic functions by rational interpolation, Proc. Colloq. Complex Analysis at Joensuu, Lecture Notes in Math., vol. 747, SpringerVerlag, Berlin, 1979, pp. 434-450.

Department of Mathematics, University of The Witwatersrand, P.O. Wits 2050, JoHANNESBURG, SOUTH AFRICA

E-mail address: 036dslecosmos.wits.ac.za 